\title{
Two-dimensional ultra-small angle X-ray scattering with grating interferometry
}

\author{
P. Modregger, ${ }^{1,2, a)}$ S. Rutishauser, ${ }^{3}$ J. Meiser,${ }^{4}$ C. David, ${ }^{3}$ and M. Stampanoni ${ }^{1,5}$ \\ ${ }^{1}$ Swiss Light Source, Paul Scherrer Institut, Villigen, Switzerland \\ ${ }^{2}$ Centre d'Imagerie BioMédicale, École Polytechnique Fédérale de Lausanne, Lausanne, Switzerland \\ ${ }^{3}$ Laboratory for Micro- and Nanotechnology, Paul Scherrer Institut, Villigen, Switzerland \\ ${ }^{4}$ Institute of Micro Structure Technology, Karlsruhe Institute of Technology, Karlsruhe, Germany \\ ${ }^{5}$ Institute for Biomedical Engineering, UZH/ETH Zürich, Zürich, Switzerland
}

(Received 30 May 2014; accepted 24 June 2014; published online 16 July 2014)

It was recently established that the pixel-wise ultra-small angle $\mathrm{x}$-ray distribution can be retrieved with grating interferometry. However, in these one dimensional approaches the contrast was limited to the direction orthogonal to the structure of the line gratings. Here, we demonstrate that sensitivity in two contrast directions can be achieved by using two pairs of crossed line gratings and by adapting scan procedures and data analysis accordingly. We demonstrate the retrieval of two-dimensional scattering distributions with grating interferometry, thus overcoming the previously reported limit of seven obtainable, complementary contrasts. In addition, we give further evidence for the superiority of the signal-to-noise ratio for the dark-field contrast, if a deconvolution-based instead of the standard analysis is utilized. (C) 2014 AIP Publishing LLC. [http://dx.doi.org/10.1063/1.4890090]

Phase contrast imaging with hard $x$-ray grating interferometry (GI) (Refs. 1 and 2) has been established as an important tool for biomedical applications ${ }^{3-5}$ and wave front sensing. ${ }^{6,7}$ Typically, the setup consists of at least two line gratings (beam splitter and analyzer grating) and provides differential phase contrast (DPC) (Ref. 8) and dark-field contrast ${ }^{9}$ in the direction orthogonal to the grating structure in addition to absorption contrast. Sensitivity in two directions can be achieved by either rotating the gratings ${ }^{10,11}$ or the sample ${ }^{12}$ and scanning multiple times. Alternatively, gratings with a two-dimensional (2D) structure can be utilized to obtain 2D sensitivity, which was experimentally demonstrated without an analyzer grating ${ }^{13-15}$ and with an analyzer grating present. ${ }^{16}$ Alternative $\mathrm{x}$-ray phase contrasts techniques have also been shown to provide $2 \mathrm{D}$ contrast, e.g., edge illumination, ${ }^{17}$ analyzer-based imaging, ${ }^{18}$ or phase propagation imaging. ${ }^{19}$

In addition to the standard contrasts provided by onedimensional (1D) GI, it was further established that the angular resolved ultra-small angle x-ray scattering distribution (USAXS) can be retrieved by 1D GI. Two distinct approaches to obtain the USAXS distributions were proposed. In Ref. 20, multiple scans acquired with different inter-grating distances, model-based data fitting, and consecutive Fourier transform were utilized. Later, this approach was combined with tomography. ${ }^{21}$

Recently, we have demonstrated that the need for multiple scans and the assumption of Gaussian-shaped scattering distributions can be avoided if the appropriate data analysis (GI-USAXS) is used. ${ }^{22} \mathrm{We}$ also proposed the combination of GI-USAXS with tomography that preserves these beneficial characteristics. ${ }^{23}$ The data analysis of GI-USAXS involves the deconvolution of noisy, periodic data, which was achieved by a careful selection of the parameters for LucyRichardson deconvolution. ${ }^{24}$ The three standard contrasts provided by 1D-GI (i.e., absorption, differential phase, and dark-field) can be regarded as parameters describing the pixel-wise USAXS distribution and the following relations were found: ${ }^{22}$ absorption corresponds to the 0th moment of the USAXS distribution, DPC to the 1st moment, and darkfield to the 2nd moment. Thus, GI-USAXS can also be used as an alternative data analysis for obtaining the standard contrasts and it was shown that GI-USAXS provides a superior signal-to-noise ratio (SNR) for the dark-field contrast. ${ }^{25}$

In this Letter, we combine the benefits of GI-USAXS with 2D-GI by utilizing two pairs of crossed line gratings (Fig. 1). The experiment was carried out at the beamline for TOmographic Microscopy and Coherent rAdiology experimenTs (TOMCAT) of the Swiss Light Source of the Paul Scherrer Institute (Villigen, Switzerland). A photon energy of $25 \mathrm{keV}$ was selected by a double multilayer monochromator, and the sample, a knot in a nylon thread, was placed at $25 \mathrm{~m}$ distance from the source. The beam splitter unit was mounted on a 2D Piezo actuator to allow for lateral positioning with $\mathrm{nm}$ precision. Each beam splitter grating

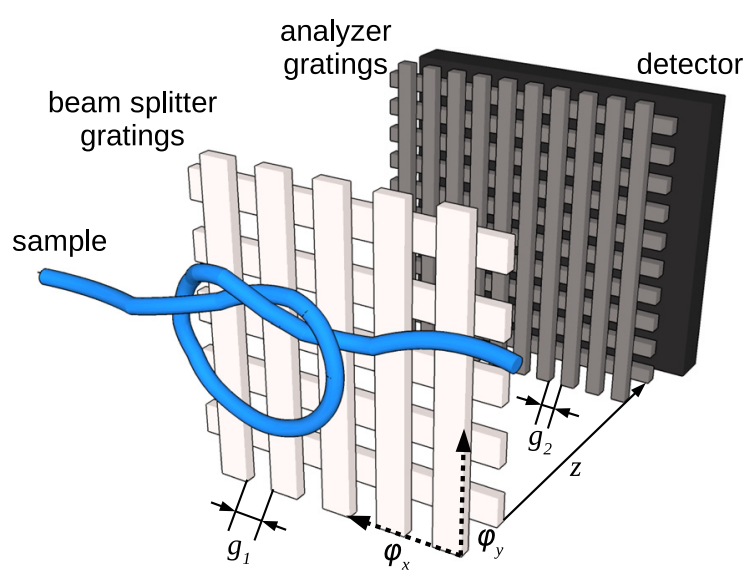

${ }^{a)}$ Electronic mail: peter.modregger@psi.ch

FIG. 1. Experimental setup for 2D GI-USAXS. 
(material: Si, pitch: $g_{1}=4.767 \mu \mathrm{m}$, and structure height: $32 \mu \mathrm{m}$ ) introduced a phase shift of $\pi$ into the beam, which realized a checkerboard-type $2 \mathrm{D}$ phase grating and provided a mesh-like interference pattern at an inter-grating distance of $z=174 \mathrm{~mm} .{ }^{26}$ At this position, the analyzer grating (material: $\mathrm{Au}$, pitch: $g_{2}=2.4 \mu \mathrm{m}$, and structure height: $50 \mu \mathrm{m}$ ) was placed directly in front of the detector unit, which had a pixel size of $7.4 \mu \mathrm{m}$ (pco.2000, PCO AG, Kelheim, Germany). The beam splitter gratings were manufactured by the Laboratory for Micro- and Nanotechnology ${ }^{27}$ of the Paul Scherrer Institute (Villigen, Switzerland), while the analyzer gratings were produced by the Institute of Micro Structure Technology of the Karlsruhe Institute of Technology (Karlsruhe, Germany). ${ }^{28}$

The typical scan procedure for 1D GI involves a lateral scan of one of the line gratings by equally distant fractions of the grating's pitch. ${ }^{6}$ This phase stepping method yields an oscillatory, periodic intensity pattern for each detector pixel, which is called a phase stepping curve (PSC). The extension to two dimensions is straightforward: $2 \mathrm{D}$ raster-like scans of the beam splitter unit in both lateral beam directions ${ }^{16}$ (indicated by the arrows in Fig. 1). Performing this scan twice, once with a sample present in the beam and once without, provides $2 \mathrm{D}$ intensity patterns $s\left(x, y, \phi_{x}, \phi_{y}\right)$ with the sample and $f\left(x, y, \phi_{x}, \phi_{y}\right)$ without the sample for each pixel $x, y$. The lateral offsets of the beam splitter unit in horizontal $\Delta_{x}$ and in vertical $\Delta_{y}$ direction are expressed as $\phi_{x, y}=2 \pi \Delta_{x, y} / g_{2}$. Here, we acquired $16 \times 16$ phase steps over one period with four accumulations for noise reduction and $150 \mathrm{~ms}$ exposure time for each frame. The resulting 2D PSCs outside and inside the sample are exemplified in Figs. 2(a) and 2(b).

For the 1D case, the scattering distribution $g(\phi)$ was implicitly defined by the convolution $s(\phi)=g(\phi) \otimes f(\phi){ }^{22}$ Again, the extension to two dimensions is straightforward yielding

$$
s\left(x, y, \phi_{x}, \phi_{y}\right)=g\left(x, y, \phi_{x}, \phi_{y}\right) \otimes f\left(x, y, \phi_{x}, \phi_{y}\right)
$$

with a $2 \mathrm{D}$ convolution with respect to $\phi_{x}$ and $\phi_{y}$. Thus, the 2D USAXS distribution $g\left(x, y, \phi_{x}, \phi_{y}\right)$ can be obtained by a pixel-wise $2 \mathrm{D}$ deconvolution of the sample and the flat PSCs. We utilized Lucy-Richardson deconvolution with 250 iterations, which took approximately $4 \mathrm{~h}$ on a modern desktop PC with 4 cores to process the entire data set consisting of $1800 \times 500 \times 16 \times 16$ data points. Examples for the retrieved 2D USAXS distributions are shown in Fig. 2(c) in an area outside the sample and in Fig. 2(d) at the edge of the sample (corresponding pixel positions are indicated by black circles in Fig. 3). Ideally, $g\left(\phi_{x}, \phi_{y}\right)$ outside of the sample should have the form of Dirac's $\delta$-distribution and deviation from this ideal (Fig. 2(c)) are due to the angular response function of the utilized data analysis procedure. The increased width of $g\left(\phi_{x}, \phi_{y}\right)$ at the edge of the sample (Fig. 2(d)) accounts for the smoothed PSC in Fig. 2(b), which demonstrates the consistency of the proposed approach.

Fig. 3 shows a montage of the retrieved scatter images of the nylon sample. Individual scatter images present $g\left(x, y, \alpha_{x}, \alpha_{y}\right)$ as it varies over the field of view for a constant angular vector $\left(\alpha_{x}, \alpha_{y}\right)$ with the refraction angles $\alpha_{x, y}=\phi_{x, y} g_{2} /(2 \pi z){ }^{8}$ The angular step from image to image is $0.9 \mu \mathrm{rad}$ in both directions.
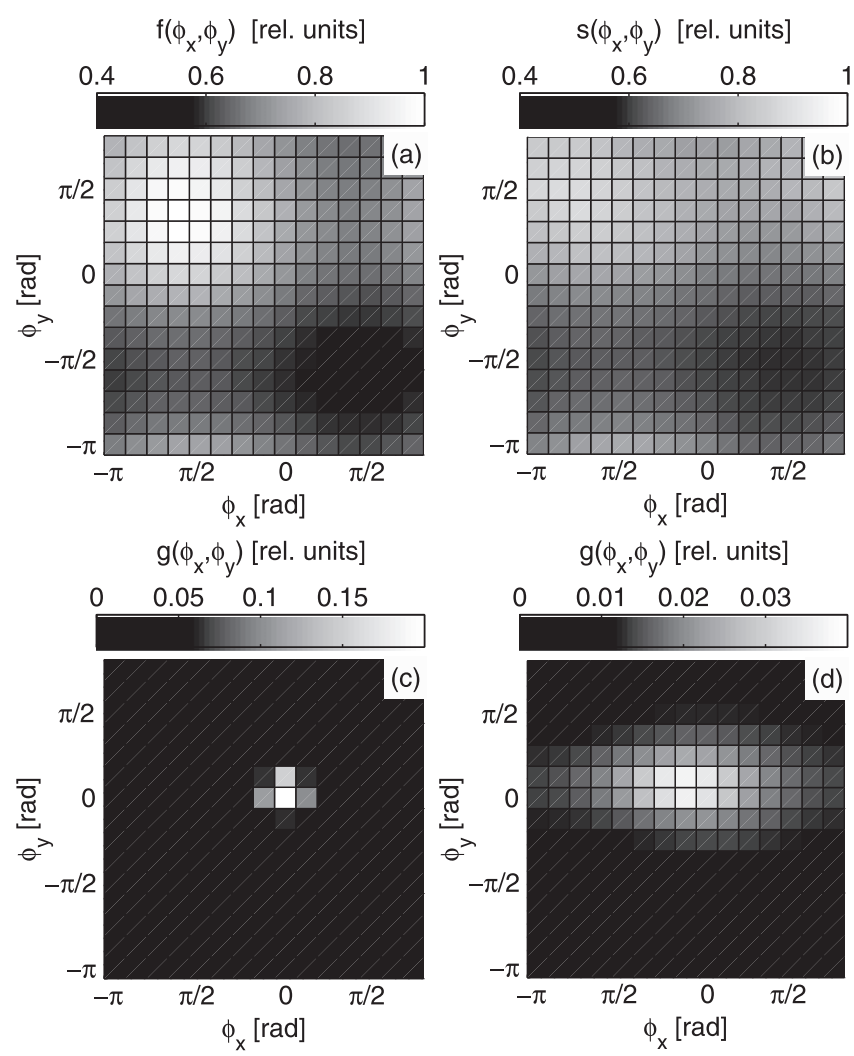

FIG. 2. Deconvolution procedure for 2D GI-USAXS. (a) and (b) are the 2D PSCs without, $f\left(\phi_{x}, \phi_{y}\right)$, and with, $s\left(\phi_{x}, \phi_{y}\right)$, the sample present in the beam. (c) and (d) are the retrieved scattering distributions, $g\left(\phi_{x}, \phi_{y}\right)$, at the locations outside and at the edge of the sample as indicated by the circles in Fig. 3. In (c), $g$ outside the sample shows a $\delta_{D}$-like shape, which meets the expectation of basically deconvolving a function with itself. In (d), the broadening of $g$ accounts for the increased scattering at the edge of the sample as expected.
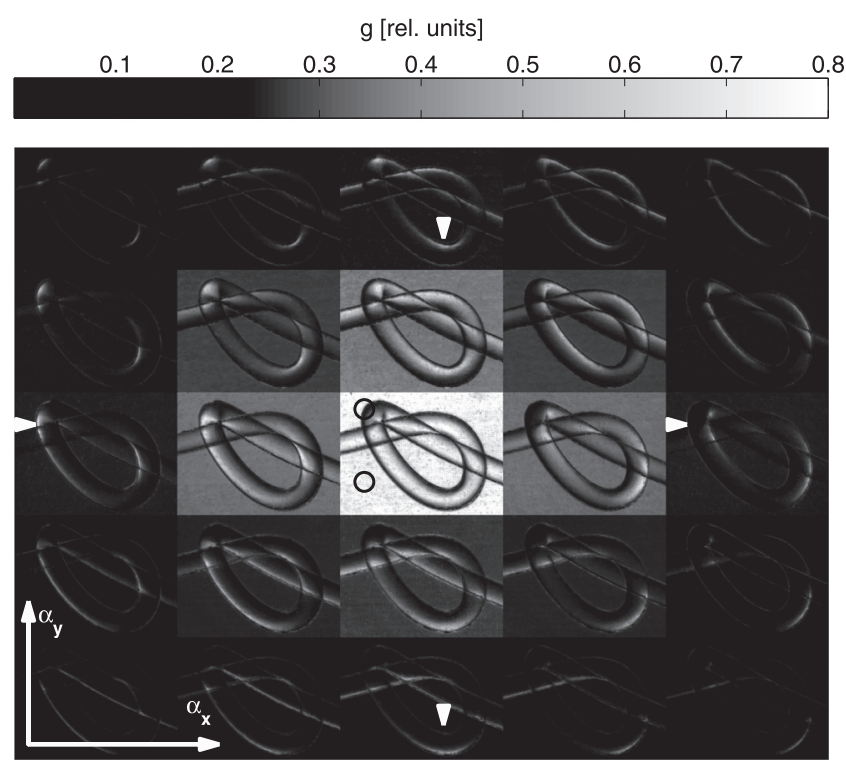

FIG. 3. Montage of scatter images of a knotted nylon thread as retrieved by 2D GI-USAXS. Each scatter image is positioned according to its angular vector $\left(\alpha_{x}, \alpha_{y}\right)$ as illustrated by the coordinate system. Out of the $16 \times 16$ available complementary contrasts the central $5 \times 5$ are shown. Triangles mark an inversion of contrast at the edge of the thread in both horizontal and vertical directions, which demonstrates $2 \mathrm{D}$ sensitivity as expected. The circles in the central image indicate the spatial positions (inside and outside of the sample) of the data used in Fig. 2. 
White triangles mark an inversion of contrast at the sample edges in both horizontal and vertical direction, which is in agreement with the expectations for 2D scattering.

In Ref. 16, the Fourier component analysis (FCA), which provides the three standard contrast in $1 \mathrm{D}$, was adapted for the 2D case. A pixel-wise 2D Fourier transform with respect to $\phi_{x}$ and $\phi_{y}$ of the PSCs yields $\hat{s}\left(x, y, q_{n}, q_{m}\right)$ and $\hat{f}\left(x, y, q_{n}, q_{m}\right)$ with $q_{n}$ and $q_{m}$ the Fourier harmonics corresponding to the horizontal and vertical direction. From here on, we suppress the dependencies on the pixel position $x, y$ in all equations for easier readability. The different contrasts modalities are retrieved by the following definitions:

$$
\begin{gathered}
\text { absorption : } A=\hat{s}(0,0) / \hat{f}(0,0), \\
\text { hor. DPC : } P_{x}=\arg \left[\hat{s}\left(q_{1}, 0\right)\right]-\arg \left[\hat{f}\left(q_{1}, 0\right)\right], \\
\text { ver. DPC : } P_{y}=\arg \left[\hat{s}\left(0, q_{1}\right)\right]-\arg \left[\hat{f}\left(0, q_{1}\right)\right], \\
\text { hor. scatter str. : } S_{x}=-2 \log \left(\left|\hat{s}\left(q_{1}, 0\right)\right| /\left|\hat{f}\left(q_{1}, 0\right)\right| A^{-1}\right), \\
\text { ver. scatter str. : } S_{y}=-2 \log \left(\left|\hat{s}\left(0, q_{1}\right)\right| /\left|\hat{f}\left(0, q_{1}\right)\right| A^{-1}\right) .
\end{gathered}
$$

The last two entries refer to the horizontal and vertical scatter strength, which are related to the dark-field contrast $B_{x, y}$ via $S_{x, y}=-2 \log B_{x, y} .{ }^{29}$ In combination with the two diagonal scatter contrasts reported in Ref. 16, the listed quantities make up the seven complementary contrasts of the standard analysis (i.e., 2D FCA). A 2D moment analysis of the retrieved scattering distributions $g\left(\phi_{x}, \phi_{y}\right)$ will be used to compare the results of GI-USAXS to the standard contrasts. For this, we define the 0th moment $M_{0}$ and the first moments in both directions $\mu_{x}$ and $\mu_{y}$ according to

$$
\begin{aligned}
M_{0} & =\int d \phi_{x} d \phi_{y} g\left(\phi_{x}, \phi_{y}\right) \\
\mu_{x, y} & =\int d \phi_{x} d \phi_{y} \phi_{x, y} g\left(\phi_{x}, \phi_{y}\right) / M_{0},
\end{aligned}
$$

and the higher order, centralized moments as

$$
M_{n m}=\int d \phi_{x} d \phi_{y}\left(\phi_{x}-\mu_{x}\right)^{n}\left(\phi_{y}-\mu_{y}\right)^{m} g\left(\phi_{x}, \phi_{y}\right) / M_{0}
$$

with the natural numbers $m>1$ and $n>1$. The PSCs were acquired in the interval $\phi_{x, y} \in[-\pi, \pi]$ and in order to account for this asymmetric interval, we symmetrized the scattering distributions according to $g\left(\phi_{x}+\pi, \phi_{y}+\pi\right)=g\left(\phi_{x}, \phi_{y}\right)$.

Fig. 4 compares the standard contrasts modalities to the corresponding moments of the scattering distributions for the knot sample. An excellent visual agreement is found for all contrasts. Thus, 2D GI-USAXS can be regarded as an alternative to 2D FCA for data analysis. We used the standard deviation, std, within a $50 \times 50$ pixel wide region of interest located in the background of the images to determine the performance of 2D FCA and 2D GI-USAXS. While we found negligible difference for the absorption $\left(\operatorname{std}(A) / \operatorname{std}\left(M_{0}\right)=1.00\right)$ and the DPC contrasts $\left(\operatorname{std}\left(P_{x}\right) / \operatorname{std}\left(\mu_{x}\right)=1.03, \operatorname{std}\left(P_{y}\right) / \operatorname{std}\left(\mu_{y}\right)\right.$ $=1.08), 2 \mathrm{D}$ GI-USAXS outperformed 2D FCA for the scattering strengths $\left(\operatorname{std}\left(S_{x}\right) / \operatorname{std}\left(\sqrt{M_{20}}\right)=4.25, \operatorname{std}\left(S_{y}\right) / \operatorname{std}\left(\sqrt{M_{02}}\right)\right.$
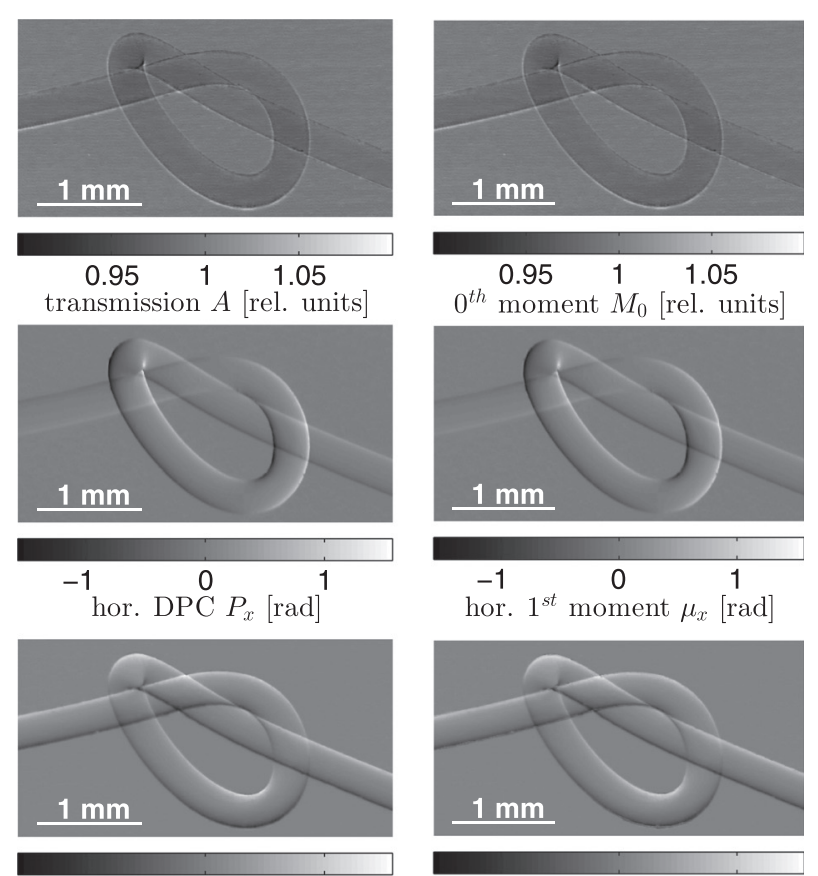

hor. $1^{\text {st }}$ moment $\mu_{x}[\mathrm{rad}]$
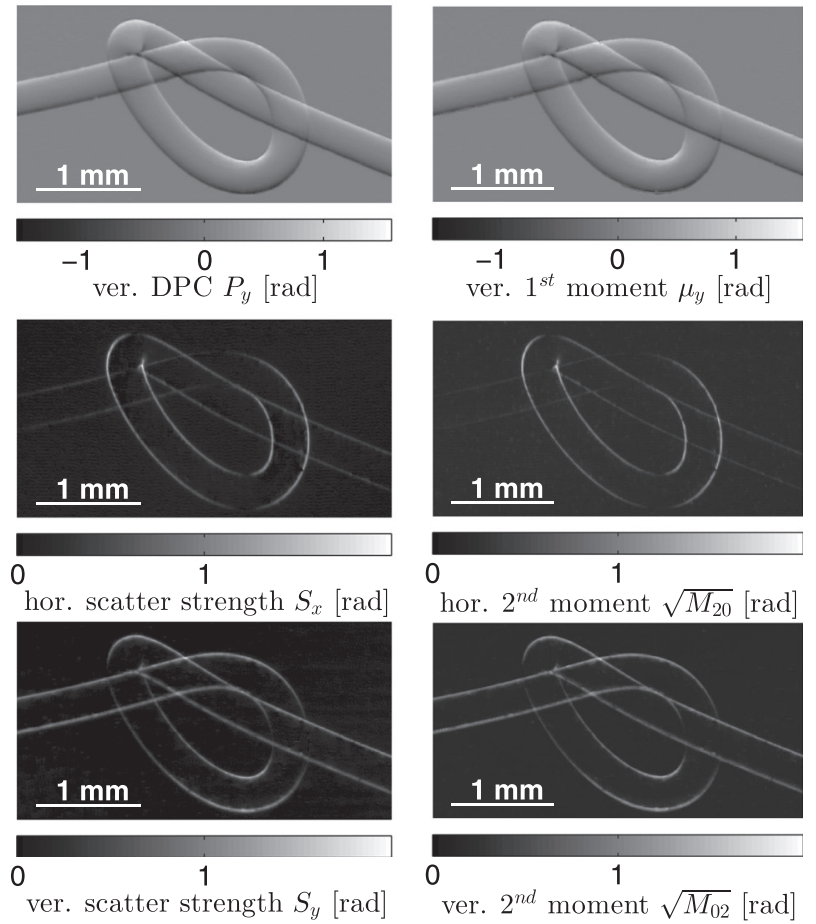

ver. $1^{\text {st }}$ moment $\mu_{y}[\mathrm{rad}]$
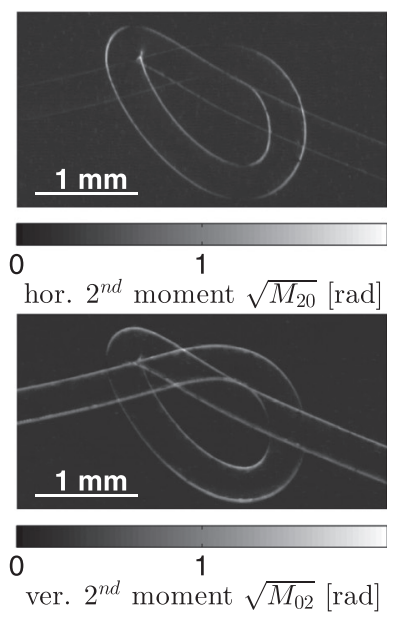

FIG. 4. Comparison of standard contrasts (left column) to the moments of the scatter distributions as retrieved by 2D GI-USAXS (right column).

$=4.38)$. Thus, we conclude that the superiority of GI-USAXS as used for the retrieval of the scatter strength over FCA that was previously reported on for the $1 \mathrm{D}$ case,${ }^{25}$ holds also true in the $2 \mathrm{D}$ case.

In addition, the horizontal third $M_{30}$ and fourth moment $M_{40}$ of the scattering distributions of the sample are displayed in Fig. 5. The third moment relates to the skew of the scattering distributions, and positive values mean that the positive tail is longer than the negative (vice versa for negative values). The fourth moment corresponds to the kurtosis, which quantifies the weight of the tails compared to the central peak. These higher order moments are not accessible by the standard data analysis approach, which shows the additional information provided by 2D GI-USAXS.

In conclusion, we extended GI-USAXS to two contrast dimensions by utilizing two pairs of crossed line gratings and by adapting scan procedures as well as data analysis. This provided the 2D ultra-small $\mathrm{x}$-ray angle scattering distributions for each pixel (i.e., 4D data sets) and increased 

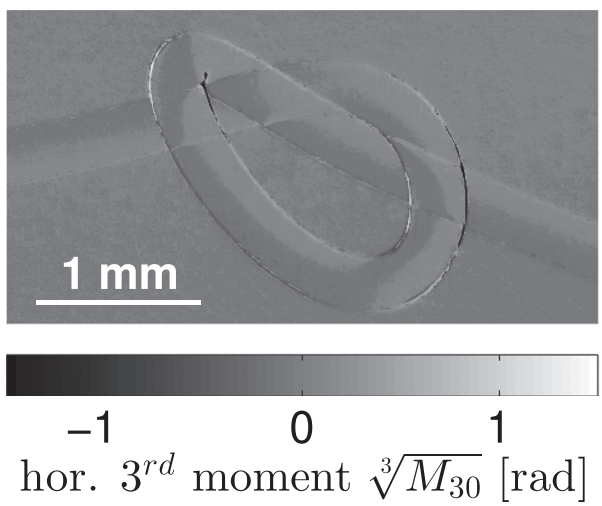
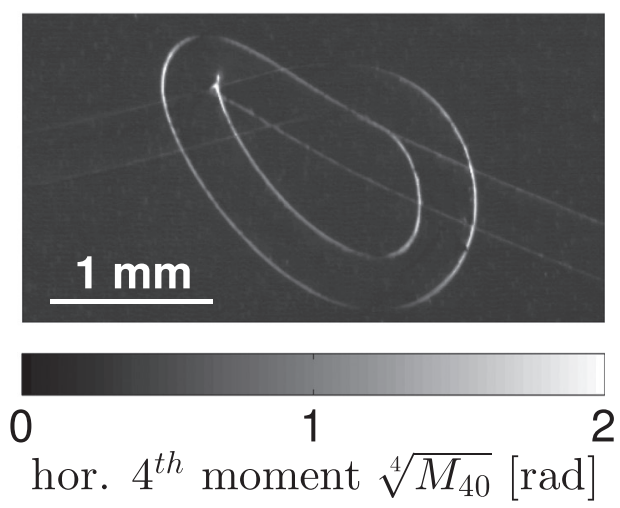

FIG. 5. Horizontal third and fourth moment of the scattering distributions demonstrating previously inaccessible information.

the number of obtainable contrasts from previously seven to $16 \times 16$ (and more). A consecutive moment analysis of scattering distributions established that GI-USAXS can be used as an alternative approach to data analysis for 2D GI and it was demonstrated that 2D GI-USAXS delivers superior noise characteristics over the established data analysis for the scatter strength images (i.e., dark-field contrast). Further, we showed that higher order moments of the scattering distributions are provided by 2D GI-USAXS, which are not accessible by the previously established data analysis.

We would like to acknowledge Gordan Mikuljan (Paul Scherrer Institut, Villigen, Switzerland) for his support on the mechanical design and Irene Zanette (Technische Universität München, Garching, Germany) for the fruitful discussions. This study was supported by Centre d'Imagerie BioMédicale (CIBM) of the UNIL, UNIGE, HUG, CHUV, and EPFL and the Leenaards and Jeantet Foundations. This work was partly carried out with the support of the Karlsruhe Nano Micro Facility (KNMF), a Helmholtz Research Infrastructure at Karlsruhe Institute of Technology (KIT).

${ }^{1}$ C. David, B. Nöhammer, H. H. Solak, and E. Ziegler, Appl. Phys. Lett. 81, 3287 (2002).

${ }^{2}$ A. Momose, S. Kawamoto, I. Koyama, Y. Hamaishi, K. Takai, and Y. Suzuki, Jpn. J. Appl. Phys., Part 2 42, L866 (2003).

${ }^{3}$ M. Stampanoni, Z. Wang, T. Thüring, C. David, E. Roessl, M. Trippel, R. A. Kubik-Huch, G. Singer, M. K. Hohl, and N. Hauser, Invest. Radiol. 46, 801 (2011).

${ }^{4}$ J. Tanaka, M. Nagashima, K. Kido, Y. Hoshino, J. Kiyohara, C. Makifuchi, S. Nishino, S. Nagatsuka, and A. Momose, Z. Med. Phys. 23, 222 (2013).

${ }^{5}$ S. Schleede, F. G. Meinel, M. Bech, J. Herzen, K. Achterhold, G. Potdevina, A. Malecki, S. Adam-Neumair, S. F. Thieme, F. Bamberg, K. Nikolaou, A. Bohla, A. Ö. Yildirim, R. Loewen, M. Gifford, R. Ruth, O. Eickelberg, M. Reiser, and F. Pfeiffer, Proc. Natl. Acad. Sci. U.S.A. 109, 17880 (2012).

${ }^{6}$ T. Weitkamp, B. Nöhammer, A. Diaz, C. David, and E. Ziegler, Appl. Phys. Lett. 86, 054101 (2005).
${ }^{7}$ S. Rutishauser, L. Samoylova, J. Krzywinski, O. Bunk, J. Grünert, H. Sinn, M. Cammarata, D. M. Fritz, and C. David, Nat. Commun. 3, 947 (2012).

${ }^{8}$ T. Weitkamp, A. Diaz, C. David, F. Pfeiffer, M. Stampanoni, P. Cloetens, and E. Ziegler, Opt. Express 13, 6296 (2005).

${ }^{9}$ F. Pfeiffer, M. Bech, O. Bunk, P. Kraft, E. F. Eikenberry, and C. David, Nat. Mater. 7, 134 (2008).

${ }^{10}$ C. Kottler, C. David, F. Pfeiffer, and O. Bunk, Opt. Express 15, 1175 (2007).

${ }^{11}$ S. Rutishauser, T. Donath, C. David, F. Pfeiffer, F. Marone, P. Modregger, and M. Stampanoni, Opt. Express 19, 24890 (2011).

${ }^{12}$ T. H. Jensen, M. Bech, O. Bunk, T. Donath, C. David, R. Feidenhans'l, and F. Pfeiffer, Phys. Med. Biol. 55, 3317 (2010).

${ }^{13}$ K. S. Morgan, P. Modregger, S. C. Irvine, S. Rutishauser, V. A. Guzenko, M. Stampanoni, and C. David, Opt. Lett. 38, 4605 (2013).

${ }^{14}$ K. L. Baker, Opt. Eng. 48, 086501 (2009).

${ }^{15}$ H. H. Wen, E. E. Bennett, R. Kopace, A. F. Stein, and V. Pai, Opt. Lett. 35, 1932 (2010).

${ }^{16}$ I. Zanette, T. Weitkamp, T. Donath, S. Rutishauser, and C. David, Phys. Rev. Lett. 105, 248102 (2010).

${ }^{17}$ A. Olivo, S. E. Bohndiek, J. A. Griffiths, A. Konstantinidis, and R. D. Speller, Appl. Phys. Lett. 94, 044108 (2009).

${ }^{18}$ P. Modregger, D. Lübbert, P. Schäfer, and R. Köhler, Appl. Phys. Lett. 90, 193501 (2007).

${ }^{19}$ P. Cloetens, W. Ludwig, J. Baruchel, D. Van Dyck, J. Van Landuyt, J.-P. Guigay, and M. Schlenker, Appl. Phys. Lett. 75, 2912 (1999).

${ }^{20}$ W. Yashiro, Y. Terui, K. Kawabata, and A. Momose, Opt. Express 18, 16890 (2010).

${ }^{21}$ W. Yashiro, S. Harasse, H. Kuwabara, K. Kawabata, and A. Momose, AIP Conf. Proc. 1466, 211 (2012).

${ }^{22}$ P. Modregger, F. Scattarella, B. R. Pinzer, C. David, R. Bellotti, and M. Stampanoni, Phys. Rev. Lett. 108, 048101 (2012).

${ }^{23}$ P. Modregger, M. Kagias, S. Peter, M. Abis, V. A. Guzenko, C. David, and M. Stampanoni, "Multiple Scattering Tomography," Phys. Rev. Lett. (in press).

${ }^{24}$ F. Scattarella, S. Tangaro, P. Modregger, M. Stampanoni, L. De Caro, and R. Bellotti, Physica Med. 29, 478 (2013).

${ }^{25}$ T. Weber, G. Pelzer, F. Bayer, F. Horn, J. Rieger, A. Ritter, A. Zang, J. Durst, G. Anton, and T. Michel, Opt. Express 21, 18011 (2013).

${ }^{26}$ I. Zanette, C. David, S. Rutishauser, and T. Weitkamp, AIP Conf. Proc. 1221, 73 (2010).

${ }^{27}$ C. David, J. Bruder, T. Rohbeck, C. Grünzweig, C. Kottler, A. Diaz, O. Bunk, and F. Pfeiffer, Microelectron. Eng. 84, 1172 (2007).

${ }^{28}$ J. Mohr, T. Grund, D. Kunka, J. Kenntner, J. Leuthold, J. Meiser, J. Schulz, and M. Walter, AIP Conf. Proc. 1466, 41 (2012).

${ }^{29}$ Z. Wang, K.-J. Kang, Z.-F. Huang, and Z.-Q. Chen, Appl. Phys. Lett. 95, 094105 (2009). 